\title{
Effect of Nicotine and Porphyromonas gingivalis on the Differentiation Properties of Periodontal Ligament Fibroblasts
}

\author{
Naruemon Panpradit ${ }^{1}$ Thanapoj Nilmoje ${ }^{1} \quad$ Julalux Kasetsuwan ${ }^{1,2} \quad$ Sujiwan Seubbuk Sangkhamanee ${ }^{1}$ \\ Rudee Surarit ${ }^{3}$ \\ ${ }^{1}$ Department of Oral Medicine and Periodontology, Faculty of \\ Dentistry, Mahidol University, Bangkok, Thailand \\ ${ }^{2}$ Section of Periodontology, College of Dental Medicine, Rangsit \\ University, Patumthani, Thailand \\ ${ }^{3}$ Department of Oral Biology, Faculty of Dentistry, Mahidol \\ University, Bangkok, Thailand

\begin{abstract}
Address for correspondence Rudee Surarit, PhD, Department of Oral Biology, Faculty of Dentistry, Mahidol University, Bangkok 10400, Thailand (e-mail: rudee.sur@mahidol.ac.th).
\end{abstract} \\ Eur J Dent 2021;15:727-732.
}

\begin{abstract}
Keywords

- cell differentiation

- nicotine

- periodontal ligament fibroblast

- Porphyromonas gingivalis
\end{abstract}

Objectives This study aimed to evaluate the effect of Porphyromonas gingivalis and nicotine on the in vitro osteogenic differentiation of periodontal ligament (PDL) fibroblasts.

Materials and Methods PDLs were cultured in Dulbecco's modified Eagle's medium containing $10 \%$ fetal bovine serum at $37^{\circ} \mathrm{C}$ under $5 \% \mathrm{CO}_{2}$ and $100 \%$ humidified atmosphere. Cells were incubated with various concentrations of nicotine and $P$. gingivalis extracts, and cell viability was determined by 3-(4,5-dimethylthiazol-2-yl)-2,5-diphenyl tetrazolium bromide assay. To study cell differentiation, PDLs $\left(5 \times 10^{4}\right.$ cells $)$ were treated with the osteogenic differentiation medium containing $10 \mathrm{mM} \beta$-glycerophosphate, $10 \mathrm{nM}$ dexamethasone, $50 \mathrm{mg} / \mathrm{mL}$ ascorbic acid, $1 \mu \mathrm{M}$ nicotine, and $50 \mu \mathrm{g} / \mathrm{mL} P$. gingivalis lysate. mRNA samples were collected at 0,7 , and 14 days. Odontogenic-related gene expression, namely, Runt-related transcription factor 2 (Runx2), collagen type I (COL1A1), and alkaline phosphatase (ALP) was determined by reverse transcription quantitative polymerase chain reaction (RT-qPCR). Calcified nodule formation was determined on day 28 using Alizarin Red S. Analysis of variance and Tukey's test were used to compare the difference among groups at significant level of $p<0.05$.

Results It showed that $50 \mu \mathrm{g} / \mathrm{mL}$ of $P$. gingivalis lysate and $1 \mu \mathrm{M}$ of nicotine showed no toxicity to PDLs. Runx2, COL1A1, and ALP expression were found to decrease significantly after 7 days of treatment, while osteocalcin expression was found to decrease after 14 days. The nodule formation in the control group was much greater in both number and size of nodules than in experimental groups, which implied a positive sign of calcium deposition in controls.

Conclusion The results indicated that nicotine and $P$. gingivalis showed adverse effect on osteogenic differentiation properties of PDLs. published online July 30,2021
DOI https://doi.org/

$10.1055 / \mathrm{s}-0041-1729678$ ISSN 1305-7456
(C) 2021. European Journal of Dentistry.

This is an open access article published by Thieme under the terms of the Creative Commons Attribution-NonDerivative-NonCommercial-License, permitting copying and reproduction so long as the original work is given appropriate credit. Contents may not be used for commercial purposes, or adapted, remixed, transformed or built upon. (https://creativecommons.org/licenses/by-nc-nd/4.0/). Thieme Medical and Scientific Publishers Pvt. Ltd. A-12, 2nd Floor, Sector 2, Noida-201301 UP, India 


\section{Introduction}

Smoking is clearly associated with the severity of periodontal disease, since evidence suggests that smokers showed high incidence of chronic periodontal disease and loss of clinical attachment. Smokers were four times more likely to have periodontitis, compared with nonsmokers. Current smokers have deeper probing depths, greater attachment loss, greater bone loss, and fewer teeth. ${ }^{1}$ Moreover, meta-analysis suggests that smoking has some effect on periodontal therapy, for both nonsurgical and surgical therapies. Periodontal pocket healing was slower for smoker than for nonsmoker patients. ${ }^{2}$ In addition, smoking affects periodontal regeneration therapy, and in furcation grade II, significantly decreased defect fill was observed in smokers, when using bone graft and membrane. ${ }^{3}$ Nicotine can also be detected in saliva, serum, and gingival crevice in smokers, where serum levels of nicotine after smoking the first cigarette and the second cigarette were $19 \pm 11.3 \mathrm{ng} / \mathrm{mL}(0.117 \pm 0.070 \mu \mathrm{M})$ and 22.9 $\pm 11.2 \mathrm{ng} / \mathrm{mL}(0.141 \pm 0.069 \mu \mathrm{M})$, respectively. ${ }^{4}$ A previous study has reported that concentrations of nicotine at 5 to $25 \mathrm{mM}$ show toxicity to the periodontal ligament (PDL) cell in Dulbecco's modified Eagle's medium (DMEM) media. ${ }^{5}$

Porphyromonas gingivalis has been suggested to be a key periodontopathic bacteria, found in the subgingival plaque of smokers ${ }^{6,7}$ Porphyromonas gingivalis was also shown to stimulate human dental follicle stem cells to produce cytokine, such as interleukin- 8 , which is related to inflammation. ${ }^{8}$ The components of $P$. gingivalis involved in pathogenicity consists of lipopolysaccharide (LPS), fimbria, and bacterial toxin. LPS from $P$. gingivalis could inhibit proliferation and differentiation of mesenchymal stem cells (MSCs). ${ }^{9}$ Moreover, LPS was shown to suppress the osteoblastic activity of PDL cells by promoting secretion of proinflammatory cytokine. ${ }^{10}$ In addition, subgingival microbial flora were found to have higher levels of $P$. gingivalis in smokers than in nonsmokers, but without significant difference. ${ }^{6,7}$

PDL fibroblasts are very important in tissue regeneration, since they can undergo differentiation into several types of cells including osteoblasts. Osteogenic differentiation is associated with the expression of several genes, such as collagen type I (COL1A1), alkaline phosphatase $(A L P)$, Runt-related transcription factor 2 (Runx2), and osteocalcin (OCN). COL1A1 is an important component of bone extracellular matrix, forming connections with cell surface integrins and other extracellular matrix proteins. ${ }^{11}$ It also plays a role in cell adhesion, proliferation, and differentiation of the osteoblast phenotype. Runx2 is a major regulator of osteoblast differentiation. ALP was reported to act as an early indicator of osteoblast activity during early osteoblast. Levels of $A L P$ mRNA have been shown to increase within 2 days after stimulation, followed by steady increases with the progression of osteoblastic differentiation, up to 14 days. ${ }^{12}$ The $O C N$ gene is one of the few osteoblast-specific genes, and encodes $O C N$, one of the most abundant proteins present in bone. ${ }^{13}$ It plays an important role in the differentiation of osteoblast progenitor cells, and shows significant upregulation in both matrix synthesis and mineralization. Differentiation of PDL cells was found to be associated with the expression of COL1A1, ALP, Runx2, and $O C N .{ }^{13}$ Nicotine also significantly decreased gene expression of $A L P, O C N$, and Runx2 during the osteogenic induction of the PDL stem cell. ${ }^{14}$

Currently, there is no information on the interaction between $P$. gingivalis and nicotine on the differentiation potential of PDL stem cells. Therefore, the present in vitro study was aimed at examining the osteogenic properties of PDL cells in the presence and absence of periodontal pathogens, with and without nicotine, under physiological conditions. We hope that this knowledge will be useful for the treatment of periodontitis in smoking patients, as well as to explain why periodontitis patients should refrain from smoking.

\section{Materials and Methods}

\section{PDL Cell Culture}

Human PDL cells from Science Cell Research Laboratories (Cat \#2630; Science Cell Research Laboratories, Carlsbad, California, United States) were used in this study. Human PDL cells were cultured in DMEM (Gibco BRL, Long Island, New York, United States) and supplemented with $10 \%$ fetal bovine serum (FBS, Hyclone, Hyclone UK Ltd., UK), and 1\% antibiotics (amphotericin B [25 $\mu \mathrm{g} / \mathrm{mL}]$, streptomycin sulphate $[10,000 \mu \mathrm{g} / \mathrm{mL}]$, and penicillin $\mathrm{G}[10,000 \mathrm{U} / \mathrm{mL}]$ ), (Hyclone, Hyclone Laboratories, Inc., Utah, United States), then incubated in $5 \% \mathrm{CO}_{2}, 37^{\circ} \mathrm{C}$, and $100 \%$ humidity. Cells at passage 4 to 6 was used for experiments.

\section{Preparation of Porphyromonas gingivalis Lysate}

Porphyromonas gingivalis (strain W50) obtained from American type culture collection (ATCC number 53978TM) was used in this study. Porphyromonas gingivalis was cultured in Trypticase soy broth in an anaerobic jar. Cells were harvested by centrifugation for 15 minutes at $2,060 \times \mathrm{g}$ at $4^{\circ} \mathrm{C}$, washed twice with PBS, and confirmed for purity by Gram staining. Cells were sonicated with high-intensity ultrasonication for 30 minutes. After that they were examined under a microscope to ensure complete cell breakdown, then centrifuged at $8,000 \times \mathrm{g}$ for 10 minutes at $4^{\circ} \mathrm{C}$ to pellet cell debris. The sample cell lysate was filtered with a filter-sterilized unit $(0.2 \mu \mathrm{m})$ and kept frozen at $80^{\circ} \mathrm{C}$ until used. Porphyromonas gingivalis lysate solution at $10,20,40$, and $50 \mu \mathrm{g} / \mathrm{mL}$ were tested on PDLs.

\section{Nicotine Preparation}

Nicotine was purchased from Sigma-Aldrich, St Louis, Missouri, United States (nicotine 99\% [TLC], liquid, N0267). Nicotine solutions was prepared into $200,100,50,25,20,10$, $1,0.1$, and $1 \mu \mathrm{M}$ in DMEM media with $10 \%$ FBS in combination with antibiotics.

\section{MTT Cytotoxicity Assay}

Cell viability was assessed by the 3-(4,5-dimethylthiazol2-yl)-2,5-diphenyl tetrazolium bromide (MTT) method. ${ }^{15}$ The PDLs from a $75-\mathrm{mm}^{2}$ flask at around $90 \%$ confluence were washed with phosphate buffered saline (1X PBS). Five mL 
of $0.25 \%$ trypsin was added, and incubated in appropriate condition $\left(5 \% \mathrm{CO}_{2}, 37^{\circ} \mathrm{C}\right.$, and $100 \%$ humidity) for 2 minutes, followed by addition of $10 \mathrm{~mL}$ of $10 \%$ complete DMEM. PDLs were seeded into 96-well plates (Costar, Corning, United States) at a density of $2 \times 10^{4}$ cells/well then treated with various concentrations of nicotine and $P$. gingivalis lysate. In the positive control group, cells were treated with $10 \%$ dimethyl sulfoxide (DMSO) and in the negative control group, cells were treated with DMEM alone. After culture in humidified atmosphere at $5 \% \mathrm{CO}_{2}$ at $37^{\circ} \mathrm{C}$ and $100 \%$ humidity for 24 hours, cells were washed with $1 \mathrm{X}$ PBS and incubated with $500 \mu \mathrm{L}$ of $0.5 \mathrm{mg} / \mathrm{mL}$ MTT reagent (3-(4,5-dimethylthiazol-2-yl)-2,5-diphenyltetrazolium bromide, Sigma-Aldrich, Inc., United States). After 2 hours, the sample plates were washed with $1 \mathrm{X}$ PBS and $100 \mu \mathrm{L}$ isopropanol was added to dissolve the formazan formed. The absorbance of each well was determined at a wavelength of $570 \mathrm{~nm}$ using a microplate reader spectrophotometer (Biotek, Winooski, United States). Experiments were performed three times in at least quadruplicate.

\section{Osteogenic Differentiation}

PDLs $\left(5 \times 10^{4}\right.$ cells/well) were seeded on a 24 -well plate (Costar, Corning) and grown under culture condition until approximately $90 \%$ confluency was reached. Then, cells were treated with the osteogenic differentiation medium containing $10 \mathrm{mM} \quad \beta$-glycerophosphate (Calbiochem), $10 \mathrm{nM}$ dexamethasone (Sigma-Aldrich), $50 \mathrm{mg} / \mathrm{mL}$ ascorbic acid (Sigma-Aldrich) as suggested by Sari et al ${ }^{15}$ for 14 days. For experimental groups, the osteogenic differentiation medium was supplemented with appropriate nicotine and/or $P$. gingivalis lysate at various concentrations. Changes in media were made daily, in order to maintain a constant elevated concentration of nicotine and $P$. gingivalis lysate, to represent chronic smoking. The experiment divided into eight groups:

Group 1: osteogenic differentiation containing medium

Group 2: osteogenic differentiation containing medium with P. gingivalis lysate

Group 3: osteogenic differentiation containing medium with nicotine
Group 4: osteogenic differentiation containing medium with $P$. gingivalis lysate and nicotine

Group 5: DMEM

Group 6: DMEM with P. gingivalis lysate

Group 7: DMEM with nicotine

Group 8: DMEM with P. gingivalis lysate and nicotine

\section{mRNA Isolation}

Following osteogenic differentiation treatment, mRNA samples from PDLs at 0,7 , and 14 days were collected from each well and analyzed for gene expression as described by Seubbuk et al. ${ }^{16}$ RNA was extracted using TRIzol reagent (Invitrogen; Thermo Fisher Scientific, Inc.) according to the manufacturer's instruction. Concentration and purity of RNA were determined by the ratio of A260/A280 using an Epoch microplate spectrophotometer (Biotek, Winooski, Vermont, United States). The contaminated DNA was eliminated by DNasel (Fermentas, Hanover, Maryland, United States). The mRNA was converted to cDNA by using iScript selected cDNA synthesis kits (Biorad, California, United States) at $42^{\circ} \mathrm{C}$ for 90 minutes, followed by $85^{\circ} \mathrm{C}$ for 5 minutes.

\section{Gene Expression Analysis}

Five genes were analyzed by quantitative polymerase chain reaction (qPCR) in order to evaluate the effect of nicotine and/or P. gingivalis on the osteogenic differentiation by using cDNA, as described earlier. The primers for the five genes, namely, the four target genes Runx2, ALP, OCN, COL1A1, and the control gene $(G A P D H)$ are shown in - Table 1. GAPDH was used as an internal control. The $20-\mu \mathrm{L}$ cocktail contained $10 \mu \mathrm{L}$ of $2 \times$ Maxima SYBR green master mix with ROX (Thermo Scientific, United States), $0.25 \mu \mathrm{M}$ of forward and reverse primers, and 50 ng of cDNA was used to perform qPCR analysis. Each gene group was transferred to the real-time PCR instrument (Stratagene, Santa Clara, California, United States) and subjected to 45 cycles. Comparative cycle threshold $\left(\mathrm{C}_{\mathrm{T}}\right)$ was analyzed for gene expression by normalizing with the reference gene (GAPDH) according to equation:

$\Delta \mathrm{C}_{\mathrm{T}}=\mathrm{C}_{\mathrm{T}}$ target $-\mathrm{C}_{\mathrm{T}}$ reference

$\Delta \Delta \mathrm{C}_{\mathrm{T}}=\Delta \mathrm{C}_{\mathrm{T}}$ test sample $-\Delta \mathrm{C}_{\mathrm{T}}$ control sample

For calculation of fold difference: $2^{-\Delta \Delta C_{T}}$

Table 1 Primers used and optimized conditions

\begin{tabular}{|l|l|l|l|l|}
\hline Target genes & Primer sequence & $\begin{array}{l}\text { Product } \\
\text { size (bp) }\end{array}$ & $\begin{array}{l}\text { Annealing } \\
\text { temperature }\left({ }^{\circ} \mathrm{C}\right)\end{array}$ & References \\
\hline $\begin{array}{l}\text { Bone formation } \\
\text { OCN }\end{array}$ & $\begin{array}{l}\text { Fw: 5'-ATGAGAGCCCTCACACTCCTCG-3' } \\
\text { Rw: 5'-GTCAGCCAACTCGTCACAGTCC-3' }\end{array}$ & 255 & 65 \\
\hline $\begin{array}{l}\text { Cell adhesion } \\
\text { COL1A1 }\end{array}$ & $\begin{array}{l}\text { Fw: 5'-AACCAAGGCTGCAACCTGGA-3' } \\
\text { Rw: 5'-GGCTGAGTAGGGTACACGCAGG-3' }\end{array}$ & 79 & 60 & 19 \\
\hline $\begin{array}{l}\text { Marker enzyme } \\
\text { ALP }\end{array}$ & $\begin{array}{l}\text { Fw: 5'-ACTGCAGACATTCTCAAA-3' } \\
\text { Rw: 5'-GAGTGAGTGAGTGAGCA-3' }\end{array}$ & 252 & 66 & 20 \\
\hline $\begin{array}{l}\text { Osteogenic tran- } \\
\text { scription factor } \\
\text { Runx2 }\end{array}$ & $\begin{array}{l}\text { Fw: 5'-GCCTTCAAGGTGGTAGCCC-3' } \\
\text { Rw: 5'-CGTTACCCGCCATGACAGTA-3' }\end{array}$ & 66 & 21 \\
\hline $\begin{array}{l}\text { Reference gene } \\
\text { GAPDH }\end{array}$ & $\begin{array}{l}\text { Fw: 5'-GCACCGTCAAGGCTGAGAAC-3' } \\
\text { Rw: 5'-TGGTGAAGACGCCAGTGGA-3' }\end{array}$ & 137 & 60 & 22 \\
\hline
\end{tabular}

Abbreviations: ALP, alkaline phosphatase; COL1A1, collagen type I; OCN, osteocalcin; Runx2, Runt-related transcription factor 2. 


\section{Biomineralization Assay Using Alizarin Red Staining}

On day 28, osteogenic differentiation was analyzed for calcium deposition by fixing the cells in $10 \%$ formalin and staining with Alizarin Red S staining (BDH Chemicals Ltd.).

\section{Statistical Analysis}

Statistical analysis was performed using a statistical software package (SPSS version 18). First gene expression results were analyzed for normal distribution by the KomogorovSmirnov's Test. Analysis of variance and multiple comparisons was used for parametric statistics analysis. Nonparametric analysis was performed using the Tukey's test to compare differences among groups. The statistical significance was determined at $p<0.05$.

\section{Results}

\section{MTT Cytotoxicity Assay}

The cell viability after treated with different concentrations of nicotine and different concentrations of $P$. gingivalis lysate was shown in - Fig. 1. Since \% cell viability was found to be more than $80 \%$ at $50 \mu \mathrm{g} / \mathrm{mL}$ of $P$. gingivalis lysate and $1 \mu \mathrm{M}$ of nicotine, so these concentrations were selected for further study. Cells were $100 \%$ killed by $10 \%$ DMSO.

\section{Effect of Nicotine and $\boldsymbol{P}$. gingivalis on Gene Expression} Significant decreases in gene expression were found between control versus nicotine and control versus $P$. gingivalis treated PDLs for the following genes: Runx2, COL1A1, ALP at day 7 and OCN at day 14 (-Fig. 2).

\section{Biomineralization Assay Using Alizarin Red Staining}

To evaluate the effect of nicotine and $P$. gingivalis on osteogenic differentiation, calcified nodule formation was assessed by Alizarin Red S. The control group showed higher numbers and larger size than all experimental groups (-Fig. 3). Furthermore, estimate of the number of nodules formed at $4 \times$ magnification showed that control had 33 nodules/well or 6.6 nodules $/ \mathrm{cm}^{2}, P$. gingivalis treatment had 10 nodules/well or 2 nodules $/ \mathrm{cm}^{2}$, nicotine treatment had 7 nodules/well or 3.5 nodules $/ \mathrm{cm}^{2}$, and nicotine treatment in combination with $P$. gingivalis had 2 nodules/well or 0.4 nodules $/ \mathrm{cm}^{2}$. No mineralized nodule was found in nonosteogenic media as shown in - Fig. $\mathbf{3}$.

\section{Discussion}

This study aims to study the differentiation of human PDL cells in response to nicotine and $P$. gingivalis. The conditions were selected to mimic the status of periodontal disease in smoking patients. MTT assay was used in this study since the assay has been well known to determine the cytotoxicity in cell culture system. ${ }^{23}$ MTT cytotoxicity test showed that 50\% inhibitory concentration of nicotine was $19.22 \mathrm{mM}$, which was similar to the $12.6 \mathrm{mM}$ found in a previous study. ${ }^{5}$ This study also found that $P$. gingivalis whole cell lysate at the concentration of $50 \mu \mathrm{g} / \mathrm{mL}$ was not toxic to PDLs similar to other studies. ${ }^{24,25}$
Therefore, experiments were performed at $1 \mu \mathrm{M}$ nicotine, a concentration similar to the plasma concentration, together with $50 \mu \mathrm{g} / \mathrm{mL} P$. gingivalis lysate which showed no toxicity. The experiment were divided into eight groups: group 1 to group 4 were treated with odontogenic/osteogenic differentiation media, while group 5 to group 8 cells were cultured without odontogenic/osteogenic differentiation inducers. Group 1 acted as a negative control of cell differentiation, group 2 was to determine the effect of $P$. gingivalis lysate alone on cell differentiation, group 3 was to determine the effect of nicotine alone on cell differentiation, while group 4 was to determine the effect of both nicotine together with $P$. gingivalis lysate. Group 5 showed the normal growth of PDLs in the DMEM medium without osteogenic differentiation inducer. Group 6 showed the effect of $P$. gingivalis lysate on PDLs and group 7 showed the effect of nicotine alone on PDLs in DMEM. Finally, group 8 showed the effect of both P. gingivalis together with nicotine on PDLs in nondifferentiation medium. It was clearly showed that no mineralized nodule was found in nonosteogenic/odontogenic medium, while several nodules were found in osteogenic/odontogenic medium similar to other studies. ${ }^{24}$

Osteoinduction medium containing dexamethasone, ascorbic acid, and beta-glycerophosphate was used. ${ }^{26}$ Gene expression was studied using four known osteogenic markers: Runx2, COL1A1, ALP, and OCN, comparing treatment and control groups. The results from groups 1 to 4 showed significant influence of $P$. gingivalis and nicotine on the osteogenic differentiation properties of PDL fibroblast, since the genes COL1A1, ALP, and Runx2 were decreased on day 7, while OCN gene expression was reduced on day 14 .

Collagen, more specifically collagen type 1 , is the most important and abundant organic component of the extracellularbonematrix that provides the bonestrengthandflexibility. ${ }^{27}$ When the cell binds with collagen, mitogen-activated protein kinase is activated, which then transfers signaling to the nucleus, and activates transcription factor Runx2 by a phosphorylation process. ${ }^{28} A L P$ gene has been reported to be associated with osteogenic differentiation. Runx2 expression was found at the onset of differentiation, and regulated osteoblast-specific genes. It can facilitate the convergence of numerous osteogenic signaling pathways including $O C N$ expression, which plays a key role in the differentiation of osteoblast progenitor cells.

The results from groups 1 to 4 also showed that in the presence of nicotine and/or P. gingivalis, osteogenic differentiation was inhibited, since the number and size of mineralized nodules on day 28 were reduced. The results were in good agreement with the gene expression data.

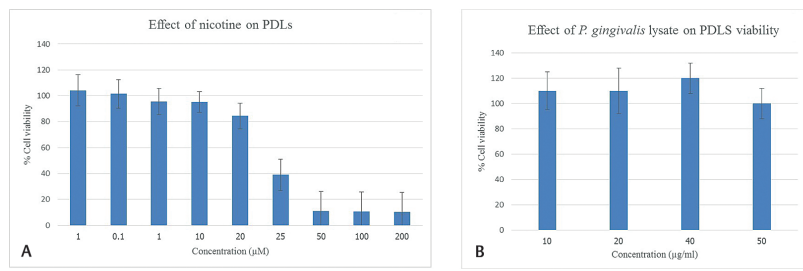

Fig. 1 Effect of nicotine and P. gingivalis lysate on cell viability (A) nicotine and (B) P. gingivalis lysate. PDLs, periodontal ligament. 


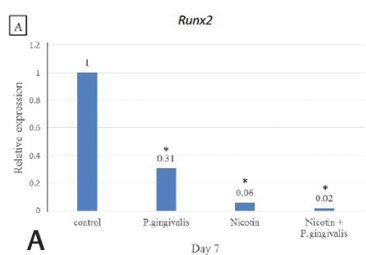

C

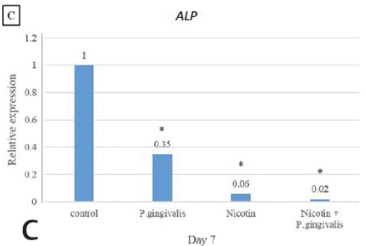

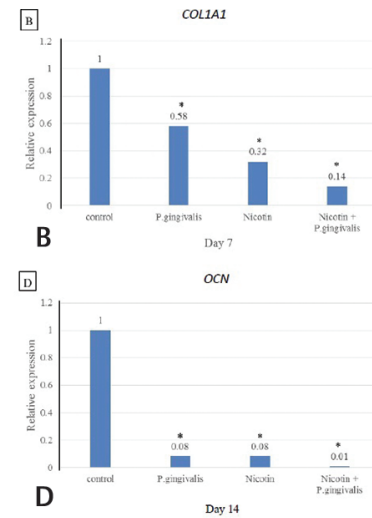

Fig. 2 The effect of nicotine and $P$. gingivalis on gene expression. The expression of Runx2 (A) COL1A1 (B), and $A L P(\mathbf{C})$ at day 7, and OCN (D) at day 14 . Statistically significant differences are shown with asterisk $\left({ }^{*}\right)$ at $p<0.05$ when compared with the control. ALP, alkaline phosphatase; COL1A1, collagen type I; OCN, osteocalcin.

Nicotine exerts its cellular functions through the nicotinic acetylcholine receptors. The subunits of the nicotinic acetylcholine receptor are present in human MSC and PDL fibroblasts. In addition, nicotine altered the microRNA (miRNA) in nicotine-treated PDL stem cell which suggested that miRNAs might play an important role in the effects of nicotine on stem cell-associated healing delay in cigarette smoking. ${ }^{29}$ A previous study reported that LPS from $P$. gingivalis could inhibit proliferation and differentiation of bone marrow stem cells. ${ }^{9}$ Porphyromonas gingivalis was also reported to affect cell proliferation and osteogenic differentiation of PDLs in vitro by downregulating Runx2 mRNA expression, and reduced mineral deposition. Other components of $P$. gingivalis such as lipid A also inhibited osteoblast function and gene expression through Toll-like receptor $2 .{ }^{11}$

Since $P$. gingivalis and nicotine have been implicated as a major etiologic factors in the development and progression of periodontitis. Periodontal pathogens and nicotine have been reported to induce oxidative stress via the endogenous superoxide mediation in PDLs. ${ }^{5}$ This study is the first to clearly show that both nicotine and $P$. gingivalis together also reduced osteogenic differentiation of PDLs, which may occur in periodontal patients who regularly smoke, possibly leading to complications in healing of the periodontium and bone during treatment. This may help explain why treatment of regular smokers for chronic periodontal disease and periodontal regeneration are rather unpredictable. These results, together with results from other experiments, confirmed the negative effects of cigarette smoke on the PDL cells and their differentiation potential, especially in the presence of periodontal pathogens. Indeed, dentists should be aware of the problem of tissue regeneration in regular smokers, and consider more frequent follow-up. The mechanisms involved in the effect of bacteria and nicotine are still unclear, but could involve oxidative stress. Our results may be applied for the treatment of periodontal disease in smoking patients and may support the recommendation for periodontal patients to stop smoking.

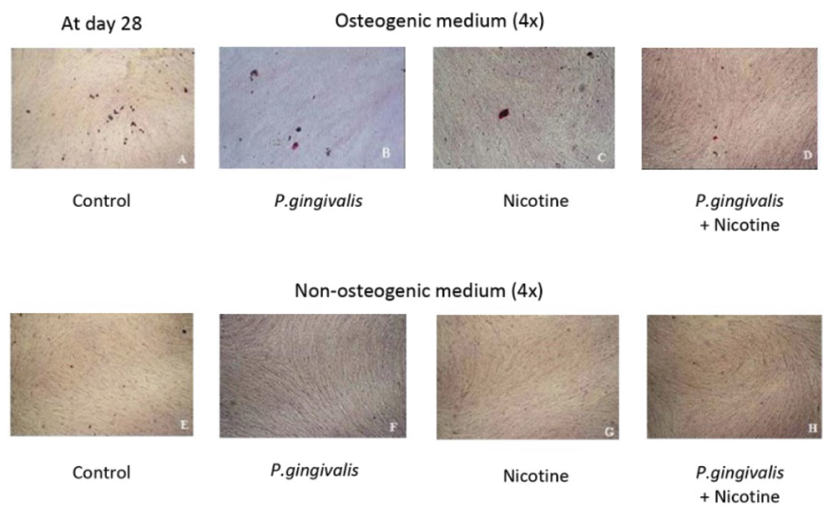

Fig. 3 The effect of nicotine and $P$. gingivalis on biomineralization on day 28. Groups 1 to 4 were grown in osteogenic media and groups 5 and 6 were grown in nonosteogenic media. (A, E) Group 1 (control), (B) group 2 (P. gingivalis lysate), (C) group 3 (nicotine), (D) group 4 (nicotine $+P$. gingivalis lysate), (F) group 5 ( $P$. gingivalis alone), (G) group 6 (nicotine alone), and $(\mathbf{H})$ nicotine $+P$. gingivalis.

\section{Conclusion}

In conclusion, this study indicated that nicotine and $P$. gingivalis showed adverse effect on osteogenic differentiation properties of PDL cells.

\section{Funding}

This work was supported by a research grant from the Faculty of Dentistry, Mahidol University.

\section{Conflict of Interest}

N.P. reported principal investigator of research grant. Rest authors declared no conflict of interest.

\section{References}

1 Ah MK, Johnson GK, Kaldahl WB, Patil KD, Kalkwarf KL. The effect of smoking on the response to periodontal therapy. J Clin Periodontol 1994;21(2):91-97

2 Labriola A, Needleman I, Moles DR. Systematic review of the effect of smoking on nonsurgical periodontal therapy. Periodontol 2000 2005;37(1):124-137

3 Bowers GM, Schallhorn RG, McClain PK, Morrison GM, Morgan R, Reynolds MA. Factors influencing the outcome of regenerative therapy in mandibular class II furcations: part I. J Periodontol 2003;74(9):1255-1268

4 Herning RI, Jones RT, Benowitz NL, Mines AH. How a cigarette is smoked determines blood nicotine levels. Clin Pharmacol Ther 1983;33(1):84-90

5 Chang YC, Huang FM, Tai KW, Yang LC, Chou MY. Mechanisms of cytotoxicity of nicotine in human periodontal ligament fibroblast cultures in vitro. J Periodontal Res 2002;37(4):279-285

6 Kinane DF, Chestnutt IG. Smoking and periodontal disease. Crit Rev Oral Biol Med 2000;11(3):356-365

7 Darby IB, Hodge PJ, Riggio MP, Kinane DF. Microbial comparison of smoker and non-smoker adult and early-onset periodontitis patients by polymerase chain reaction. J Clin Periodontol 2000;27(6):417-424

8 Biedermann A, Kriebel K, Kreikemeyer B, Lang H. Interactions of anaerobic bacteria with dental stem cells: an in vitro study. PLoS One 2014;9(11):e110616 
9 Chatzivasileiou K, Kriebel K, Steinhoff G, Kreikemeyer B, Lang $\mathrm{H}$. Do oral bacteria alter the regenerative potential of stem cells? A concise review. J Cell Mol Med 2015;19(9):2067-2074

10 Kato H, Taguchi Y, Tominaga K, Umeda M, Tanaka A. Porphyromonas gingivalis LPS inhibits osteoblastic differentiation and promotes pro-inflammatory cytokine production in human periodontal ligament stem cells. Arch Oral Biol 2014;59(2):167-175

11 Okazaki K, Sandell LJ. Extracellular matrix gene regulation. Clin Orthop Relat Res 2004;(427(Suppl):S123-S128

12 Prins HJ, Braat AK, Gawlitta D, et al. In vitro induction of alkaline phosphatase levels predicts in vivo bone forming capacity of human bone marrow stromal cells. Stem Cell Res (Amst) 2014;12(2):428-440

13 Choi MH, Noh WC, Park JW, Lee JM, Suh JY. Gene expression pattern during osteogenic differentiation of human periodontal ligament cells in vitro. J Periodontal Implant Sci 2011;41(4):167-175

14 Zhou Z, Li B, Dong Z, et al. Nicotine deteriorates the osteogenic differentiation of periodontal ligament stem cells through $\alpha 7$ nicotinic acetylcholine receptor regulating Wnt pathway. PLoS One 2013;8(12):e83102

15 Sari DS, Maduratna E, Latief FDE, Nugraha AP, Sudiana K, Rantam FA; Ferdiansyah. Satuman. Osteogenic differentiation and biocompatibility of bovine teeth scaffold with rat adipose-derived mesenchymal stem cells. Eur J Dent 2019;13(2):206-212

16 Seubbuk S, Sritanaudomchai H, Kasetsuwan J, Surarit R. High glucose promotes the osteogenic differentiation capability of human periodontal ligament fibroblasts. Mol Med Rep 2017;15(5):2788-2794

17 Mosmann T. Rapid colorimetric assay for cellular growth and survival: application to proliferation and cytotoxicity assays. J Immunol Methods 1983;65(1-2):55-63

18 Ge S, Zhao N, Wang L, Liu H, Yang P. Effects of hydroxyapatite nanostructure on channel surface of porcine acellular dermal matrix scaffold on cell viability and osteogenic differentiation of human periodontal ligament stem cells. Int J Nanomedicine 2013;8:1887-1895
19 Mah W, Jiang G, Olver D, et al. Human gingival fibroblasts display a non-fibrotic phenotype distinct from skin fibroblasts in three-dimensional cultures. PLoS One 2014;9(3):e90715

20 Jacobs C, Grimm S, Ziebart T, Walter C, Wehrbein $H$. Osteogenic differentiation of periodontal fibroblasts is dependent on the strength of mechanical strain. Arch Oral Biol 2013;58(7):896-904

21 Zhou Y, Wu C, Xiao Y. The stimulation of proliferation and differentiation of periodontal ligament cells by the ionic products from Ca7Si2P2O16 bioceramics. Acta Biomater 2012;8(6):2307-2316

22 Wang L, Wang ZH, Shen CY, You ML, Xiao JF, Chen GQ. Differentiation of human bone marrow mesenchymal stem cells grown in terpolyesters of 3-hydroxyalkanoates scaffolds into nerve cells. Biomaterials 2010;31(7):1691-1698

23 Vajrabhaya LO, Korsuwannawong S, Surarit R. Cytotoxic and the proliferative effect of cuttlefish bone on MC3T3-E1 osteoblast cell line. Eur J Dent 2017;11(4):503-507

24 Nguyen TT, Huynh NN, Seubbuk S, Nilmoje T, Wanasuntronwong A, Surarit R. Oxidative stress induced by Porphyromonas gingivalis lysate and nicotine in human periodontal ligament fibroblasts. Odontology 2019;107(2): 133-141

25 Morioka M, Hinode D, Nagata A, et al. Cytotoxicity of Porphyromonas gingivalis toward cultured human gingival fibroblasts. Oral Microbiol Immunol 1993;8(4):203-207

26 Langenbach F, Handschel J. Effects of dexamethasone, ascorbic acid and $\beta$-glycerophosphate on the osteogenic differentiation of stem cells in vitro. Stem Cell Res Ther 2013;4(5):117

27 Viguet-Carrin S, Garnero P, Delmas PD. The role of collagen in bone strength. Osteoporos Int 2006;17(3):319-336

28 Satija NK, Gurudutta GU, Sharma S, et al. Mesenchymal stem cells: molecular targets for tissue engineering. Stem Cells Dev 2007;16(1):7-23

$29 \mathrm{Ng}$ TK, Carballosa CM, Pelaez D, et al. Nicotine alters microRNA expression and hinders human adult stem cell regenerative potential. Stem Cells Dev 2013;22(5):781-790 